\title{
The Application of Image Processing in the Criminal Investigation
}

\author{
Weiyi Shi, a \\ ${ }^{1}$ School of Software Institute, Dalian Jiaotong University, Dalian 116052, China; \\ a405486928@qq.com
}

Keywords: Image processing, criminal investigation, application.

\begin{abstract}
Image processing technology plays an important role in fighting against crime and criminal cases. In the case investigation, the image processing technology can provide evidence for the trial and the litigation of the case, the case investigators can take this as a clue, and then detect the case. However, due to the external objective factors and the level of its own technology, monitoring system which is used to record video image information often appears degraded, blurred, and cannot reach the desired results. Therefore, this paper discusses the connotation of image processing technology in criminal investigation, and introduces a variety of fuzzy image processing technology, and analyses the contrast ratio enhancement, image to fog and image restoration in detail.
\end{abstract}

\section{Introduction}

The purpose of using video surveillance images to carry out the investigation is that investigators use the video monitoring data collected by themselves to get clues about the cases, then found and confirmed the identity of the suspect. The core task of image detection is to analyze video image to access to the case clues and evidence which is help to solve cases to public security organs, so as to achieve the purpose of image detection. At present, in the process of video analysis, investigators often encounter the problem that the key video footage is fuzzy, but the picture or a frame related to the features of the criminal suspect or the vehicle license plate number, so image processing technology plays an important role in solving the fuzzy video information. To this end, the application of image processing technology in criminal investigation has some guidance and reference for the actual combat department.

The common fuzzy image in monitoring system can be divided into the following several types: (1) the low contrast image caused by underexposure or overexposure; (2) the degraded image which is shoot in bad weather such as greasy weather; (3) noise blurred image; (4) motion blurred image caused by a fast-moving target; (5) the defocus blurred image caused by the lens out of focus; (6) low resolution image. In addition, because of the limitation of bandwidth and storage, the monitoring system will compress the collected video image firstly, and then transmit it to the back end monitoring platform. Compression technology can effectively reduce the bandwidth loss of the system, but it is also bound to lose some useful details. In order to obtain useful details from these fuzzy video images, it is necessary to process fuzzy image.

Fuzzy image processing technology refers to use digital image processing algorithm to process the fuzzy image, and restore or enhance the original target details, so as to obtain valuable information [1]. Because there are many kinds of reasons that cause image blurring, in order to get better results, we need to adopt a clear algorithm for different types of fuzzy images. Technically, fuzzy image processing technology is involved in many kinds of image processing and computer vision technology, such as image contrast enhancement, image defogging, image denoising, image restoration and super-resolution reconstruction.

\section{The Connotation of the Forensic Image Processing Technology}

Image processing technology refers to the technology that use a series of image processing in order to achieve the intended objectives. Image processing technology includes analog image 
processing, digital image processing and photoelectric combination processing. At present, the retrieval investigation of video surveillance image are the basic digital images, and this images can be processed by computer and in the category of digital image processing. Digital image processing includes a very rich content, according to the level of abstraction, it can be divided into three levels: the narrow sense image processing, image analysis, and image understanding.

Narrow sense image processing is one of the most common methods used in the daily image processing or in the investigation of the application. In essence, it is the process of obtaining a new matrix by the proper operation of the matrix pixel value of the image. Fuzzy image processing belongs to the category of narrow sense image processing. The handling methods of fuzzy image processing particularly are image enhancement, image restoration, and image reconstruction, and the main purpose is to restore blur image into relatively clear image, including the operation of removal of noise, rotation deformation, change the image size and resolution, adjust the brightness and contrast ratio etc.

Image analysis is based on the narrow sense image processing, and mainly focuses on the analysis of the image content. It includes static image analysis and dynamic image analysis. Static image analysis refers to the representation of turning images into numbers or symbols; dynamic image analysis refers to the process that turn related information of multi frame images or multiple dynamic image fragment into some meaningful information. For example, the extraction of characteristic value of the fingerprint image and the characteristic value of the vehicle license plate image are the process of static image analysis. The analysis for dynamic image, such as in a hotel room there was a murder case, according to site survey and investigation situation, investigators determine the crime number and the time, then extract the video surveillance data to the corresponding period of time in the hotel and the hotel to analyze and obtain evidence. In the video image analysis, the investigators can analyze video monitoring data of the gate of the hotel by digital form to describe the import number and time at first, and then do the same analysis for the video monitoring information of all and get the number and time that appeared, the number and time are valuable information.

Image understanding is the process that to deal with the image to get the result on the basis of the image analysis, and this process is similar to the human thinking process. For example, face recognition is the image understanding, that is, through the image analysis to extract the face feature value, and then to identify and compare the extracted face feature to get the recognition results. Similarly, in the given example of image analysis, it is possible to analyze and judge the suspect to compare the number and time which is analyzed by every probe of video data in hotels. And it is the process of image understanding.

\section{Common Fuzzy Image Processing Method}

There are many types of fuzzy image, in order to get better results, we need to adopt a clear algorithm. According to the number of images involved in processing, fuzzy image processing can be divided into two categories: single frame processing and sequential image processing. Contrast enhancement, image defogging and image restoration are generally only for single image operation, and they belong to a single frame processing method; but super resolution reconstruction requires the integration of multiple images information, to obtain more detailed information than a single image results, so it belongs to image sequence processing method. In order to eliminate random noise, image denoising can be achieved not only by using spatial information in a single image, but also through the time domain multi image average, that's to say, specific methods need to be determined according to the actual situation.

\subsection{Contrast Ratio Enhancement}

Contrast ratio enhancement is a necessary means to improve the visual quality of low contrast ratio images. The commonly used algorithms are Gray Scale Transformation, Histogram Processing, and Retinex Algorithm, etc.

In low contrast ratio images, the original narrow gray range is mapped to a wider range by linear or nonlinear transformation, which is referred to as the gray transformation method [2]. This 
method can enhance a lot of details of the image dark area, with the characteristics of principle of simple, easy to achieve and fast computing time, etc. The main disadvantage of the algorithm is poor adaptability, and it need to adjust the parameters of the algorithm according to the specific brightness level.

Histogram equalization is a classical point processing algorithm in computer vision and image processing, and it is very suitable for the narrow gray range of low illumination [2]. Compared with the gray level transformation, in addition to the expansion of the dynamic range, histogram equalization also can ensure the uniform distribution of gray level. As a kind of point processing algorithm, its computation is very little, the speed is fast, and it can be completed by one times of the probability statistics and mapping of the gray value of an image.

According to the color constancy theory, Land proposed Retinex Theory in the last century in 70s [3]. Random Walk Algorithm, Recursive Implementation, the Center /Surround Algorithm, the Algorithm Based On the Calculus of Variations and other algorithms have been proposed, and is widely used in color fidelity, image contrast ratio enhancement, high dynamic range compression and other image enhancement field, and achieved good results. The main difficulty of this method lies in the estimation of image illumination, which avoids the edge halo effect produced by many algorithms.

\subsection{Image Defogging}

Surveillance video images collected under bad weather (fog, haze, etc.) often exhibit low visibility, which is mainly caused by two reasons: one is the absorption and scattering of bounce light of objects which is caused by larger water particles or dust in the atmosphere, and the absorption and scattering will weak its strength; on the other hand, the light received by the sensor of camera, mixed with sky light which is caused by diffuse reflection of atmospheric particles, reducing the contrast of object. Because the degree of atmospheric absorption and scattering of light are related to the distance between the target and the camera, so the reduction of visibility of an image is changed depend on the space, which is different from the usual fuzzy and low contrast ratio images [4].

In recent years, the single image defogging algorithm based on fog imaging degradation mode has made significant progress. Because the degraded model constraints are insufficient, these algorithms typically use some assumptions or prior knowledge to estimate the transmission coefficient of the unknown, such as the dark channel prior, transmission coefficient regardless of surface color, smooth change of transmission coefficient and etc. When the transmission coefficient is estimated, the defogging method can recover clear image by using the atmospheric imaging physical model.

\subsection{Image Restoration}

Defocusing blurred image and motion blurred image are two kinds of common fuzzy forms in video surveillance. Defocusing blur is that the focal length of the lens is inaccuracy when to capture video which made the target not so clear. The character is that image fuzzy degree show the shape of the disc, especially at the point light source. Motion blur is caused by the rapid movement of the object during the shooting period. Because the exposure time of the camera is very short, it can be considered that during this period, the moving speed of the object is constant, and the movement of the whole object is the same, and the movement type is linear motion. The difference between defocusing blur and motion blur is that motion blur is only in one direction which is in the same direction as the motion. In view of these two kinds of fuzzy image, the image restoration technique is often used to get better results.

Image restoration is based on the prior knowledge of image degradation to establish a regression model, and then use this model as the basis, to restore with a variety of inverse operations, so as to achieve the purpose of restoring the image details of the information [5]. Due to the image blur caused by the out of focus, motion and other reasons, the method of image restoration is better. Although image restoration and image enhancement are all used to improve the clarity of the image and improve the quality of the image, but they have essential difference in the technology. Image enhancement does not need to know the specific degradation model and parameters of the fuzzy 
image (such as the fuzzy scale and angle of motion blur), mainly through the adjustment of the value of image brightness to enhance the visual effect of image. But image restoration should fully know a priori knowledge of the image degradation process, and then using the inverse operation, so as to get clear image. Due to the use of inverse operation, the selection of the fuzzy image degradation model and parameters directly determine the effect of image restoration.

The commonly used restoration methods include Inverse Filtering, Wiener Filtering, the Iterative Method with Constraints and etc. Wiener filter has better denoising performance, and the mean square error between the blurred image and restored image is the least, which has a wide range of applications in the actual engineering.

\section{Conclusion}

The application of computer image processing technology in the investigation of the case is also more and more extensive, so as to provide a more favorable conditions for the rapid detection of cases. In practical applications, due to various degradation factors, the fuzzy image is a bruising of video monitoring system. With the development of digital image technology and the promotion of market demand, the research of fuzzy image processing technology is developing fast. A large number of practical cases show that the fuzzy image processing technology plays an important role in vehicle license plate recognition, portrait clarity, etc., and it has great significance for the police to find clues, provide evidence and so on.

This paper analyzes the connotation of criminal investigation and several kinds of fuzzy image types for video monitoring system in common, introduced a variety of fuzzy image processing technology and the contrast enhancement, image defogging and image restoration technology in detail, and the actual processing results are also given. We believe that with the continuous development of technology, fuzzy image processing in the future will become an indispensable tool in criminal investigation and other public security areas.

\section{References}

[1]. Caponetti, L., \& Castellano, G. (2017). Fuzzy Image Processing. In Fuzzy Logic for Image Processing (pp. 53-63). Springer International Publishing.

[2]. Eberhardt, J. L., Goff, P. A., Purdie, V. J., \& Davies, P. G. (2004). Seeing black: race, crime, and visual processing. Journal of personality and social psychology, 87(6), 876.

[3]. Land, E. H., \& McCann, J. J. (1971). Lightness and retinex theory. JOSA,61(1), 1-11.

[4]. Kerre, E. E., \& Nachtegael, M. (Eds.). (2013). Fuzzy techniques in image processing (Vol. 52). Physica.

[5]. Lee, C. S., Kuo, Y. H., \& Yu, P. T. (1997). Weighted fuzzy mean filters for image processing. Fuzzy Sets and systems, 89(2), 157-180. 